\title{
The B0.5 IVe CoRoT target HD 49330
}

\section{Spectroscopic ground-based observations ${ }^{\star}$}

\author{
M. Floquet ${ }^{1}$, A.-M. Hubert ${ }^{1}$, A.-L. Huat ${ }^{1}$, Y. Frémat ${ }^{2}$, E. Janot-Pacheco ${ }^{3}$, J. Gutiérrez-Soto ${ }^{1}$, C. Neiner ${ }^{1}$, B. de Batz ${ }^{1}$, \\ B. Leroy ${ }^{4}$, E. Poretti ${ }^{5}$, P. Amado ${ }^{7}$, C. Catala ${ }^{4}$, M. Rainer ${ }^{5}$, D. Diaz ${ }^{7}$, K. Uytterhoeven ${ }^{6,5}$, L. Andrade ${ }^{7}$, P. D. Diago ${ }^{8}$, \\ M. Emilio ${ }^{9}$, F. Espinosa Lara ${ }^{1}$, J. Fabregat ${ }^{8}$, C. Martayan ${ }^{2,1}$, T. Semaan ${ }^{1}$, and J. Suso ${ }^{8}$ \\ 1 GEPI, Observatoire de Paris, CNRS, Université Paris Diderot, 5 place Jules Janssen, 92190 Meudon, France \\ e-mail: michele.floquet@obspm. fr \\ 2 Royal Observatory of Belgium, 3 avenue Circulaire, 1180 Brussels, Belgium \\ 3 Universidad de São Paulo, IAG-USP, Brazil \\ ${ }^{4}$ LESIA, Observatoire de Paris, CNRS, Université Paris Diderot, 5 place Jules Janssen, 92190 Meudon, France \\ 5 INAF - Osservatorio Astronomico di Brera, via E. Bianchi 46, 23807 Merate (LC), Italy \\ ${ }^{6}$ Laboratoire AIM,CEA/DSM-CNRS-Université Paris Diderot; CEA, IRFU, SAp, centre de Saclay, \\ 91191 Gif-sur-Yvette Cedex, France \\ 7 Instituto de Astrofísica de Andalucía (CSIC), Apartado 3004, 18080 Granada, Spain \\ 8 Observatori Astronòmic de la Universitat de València, ED. Instituts d'Investigatió, Poligon La Coma, 46980 Paterna, València, \\ Spain \\ ${ }_{9}$ Observatorio Astronomico/DEGEO, Universidade Estadual de Ponta Grossa, Brazil
}

Received 23 February 2009 / Accepted 20 May 2009

\section{ABSTRACT}

Context. We present spectroscopic ground-based observations of the early Be star HD 49330 obtained simultaneously with the CoRoT-LRA1 run just before the burst observed in the CoRoT data.

Aims. Ground-based spectroscopic observations of the early Be star HD 49330 obtained during the precursor phase and just before the start of an outburst allow us to disantangle stellar and circumstellar contributions and identify modes of stellar pulsations in this rapidly rotating star.

Methods. Time series analysis (TSA) is performed on photospheric line profiles of He I and Si III by means of the least squares method.

Results. We find two main frequencies $f 1=11.86 \mathrm{c} \mathrm{d}^{-1}$ and $f 2=16.89 \mathrm{c} \mathrm{d}^{-1}$ which can be associated with high order p-mode pulsations. We also detect a frequency $f 3=1.51 \mathrm{c} \mathrm{d}^{-1}$ which can be associated with a low order g-mode. Moreover we show that the stellar line profile variability changed over the spectroscopic run. These results are in agreement with the results of the CoRoT data analysis, as shown in Huat et al. (2009).

Conclusions. Our study of mid- and short-term spectroscopic variability allows the identification of p- and g-modes in HD 49330. It also allows us to display changes in the line profile variability before the start of an outburst. This brings new constraints for the seimic modelling of this star.

Key words. stars: early-type - stars: emission-line, Be - stars: individual: HD 49330 - stars: rotation - stars: oscillations

\section{Introduction}

Be stars are early-type stars with emission lines arising from circumstellar material (disk). They are well known to exhibit periodic and cyclic light and line-profile variations with timescales ranging from hours to decades. Be stars are located in the same domain as $\beta$ Cephei and SPB stars in the HR diagram. However, unlike these 2 groups of pulsating stars, Be stars are rapidly rotating.

For Be stars, the CoRoT light curve has to be considered as the result of the superposition of stellar and circumstellar

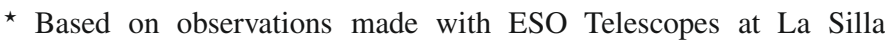
Observatory under the ESO Large Programme: LP178.D-0361, and on data collected with the TBL at Pic du Midi Observatory (France). variability effects, which involve non radial pulsations and possibly rotational modulation due to magnetic fields as well as starto-disk mass transfer and density changes in the circumstellar material as a consequence of outflow events. Emission, which affects photospheric lines and the continuum, is often highly variable in early Be stars, polluting the intrinsic stellar variability. Ground-based spectroscopy is then needed to investigate the different types of variability present in these objects.

Earlier (B0-B1) Be stars are very active (Hubert \& Floquet 1998). HD 49330 (V739 Mon, B0.5IVe) has been chosen as one of the CoRoT targets in the seismology field of the LRA1 run. This star is known to present light variability with several timescales (Gutiérrez-Soto et al. 2007). The photometric curve obtained from previous ground-based observations (ASAS and 




Fig. 1. CoRoT light curve of HD 49330. Horizontal red lines indicate the epochs of ground-based observations and the cross the BeSS observation.

OSN observations) displays a long-term variation ( $>2000$ days) with a maximum of brightness around HJD 2453 400, disrupted by several outbursts (see Fig. 1 in Huat et al. 2009, hereafter Paper I). CoRoT observations have been performed on the decreasing branch of the light curve and show an outburst of moderate intensity $(0.03 \mathrm{mag})$ offering the opportunity to observe the different phases of a light outburst (the precursor phase, the outburst itself and the beginning of the relaxation phase) (see Paper I).

Spectroscopic observations have been obtained simultaneously with the CoRoT LRA1 run. The aim of these observations is to disentangle stellar and circumstellar contributions and identify modes of stellar pulsations. This will allow an easier interpretation of the results obtained from the CoRoT light curve (Paper I).

\section{Ground-based spectroscopic observations}

HD 49330 has been monitored in the framework of a worldwide collaboration aimed to secure the spectroscopic observation of targets in the seismology field of CoRoT. The data have been acquired simultaneously with the CoRoT-LRA1 run, which started on 10 October 2007 and finished on 03 March 2008. Ground-based spectroscopic observations were first carried out from 12 to 19 December 2007 with NARVAL, a new generation spectro-polarimeter for the $2 \mathrm{~m}$ Telescope Bernard Lyot (TBL) (Pic du Midi Observatory, France) and second, from 18 to 28 December 2007 and 9 to 14 January 2008 at ESO with the FEROS spectrograph (2.2 m telescope, La Silla, Chile). A spectrum was also retrived from $\mathrm{BeSS}^{1}$.

Ground-based observations were performed just before the occurence of an outburst of moderate intensity observed in the CoRoT data (see Fig. 1). The CoRoT light curve depicts four sequences across the 138-d run: first, a rather quiescent phase; second, a pre-burst phase characterized by a weak and gradual flux decrease; third, the occurence of the outburst itself with an increase of brightness of $0.03 \mathrm{mag}$ which covers more than 50 days; fourth, the beginning of the relaxation phase, which is not complete at the end of the LRA1 CoRoT run.

\footnotetext{
1 This work has made use of the BeSS database, operated at GEPI, Observatoire de Meudon, France: http://basebe.obspm.fr
}

Table 1. Log of observations at ESO/FEROS at $2.2 \mathrm{~m}$ and NARVAL at $2.0 \mathrm{~m}$ instruments for HD 49330.

\begin{tabular}{llll}
\hline \hline Date & $N$ sp & $\begin{array}{l}\text { Span time } \\
\text { day }\end{array}$ & $S / N$ \\
\hline NARVAL & & & \\
Dec. 12-13 2007 & 4 & 0.049 & $72-110$ \\
Dec. 13-14 2007 & 8 & 0.114 & $80-120$ \\
Dec. 14-15 2007 & 8 & 0.098 & $82-120$ \\
Dec. 15-16 2007 & 8 & 0.114 & $70-87$ \\
Dec. 16-17 2007 & 8 & 0.114 & $52-88$ \\
Dec. 18-19 2007 & 6 & 0.065 & $58-103$ \\
\hline FEROS & & & \\
Dec. 18-19 2007 & 9 & 0.278 & $180-285$ \\
Dec. 19-20 2007 & 10 & 0.267 & $190-280$ \\
Dec. 20-21 2007 & 10 & 0.271 & $180-275$ \\
Dec. 21-22 2007 & 10 & 0.273 & $220-290$ \\
Dec. 22-23 2007 & 10 & 0.267 & $240-295$ \\
Dec. 23-24 2007 & 10 & 0.370 & $200-270$ \\
Dec. 24-25 2007 & 10 & 0.269 & $220-310$ \\
Dec. 25-26 2007 & 7 & 0.179 & $225-280$ \\
Dec. 26-27 2007 & - & - & - \\
Dec. 27-28 2007 & 10 & 0.264 & $195-280$ \\
Jan. 09-10 2008 & 1 & - & 144 \\
Jan. 10-11 2008 & 10 & 0.283 & $215-245$ \\
Jan. 11-12 2008 & 10 & 0.277 & $155-200$ \\
Jan. 12-13 2008 & 10 & 0.278 & $165-210$ \\
Jan. 13-14 2008 & 10 & 0.279 & $170-220$ \\
\hline BeSS & & & \\
Dec. 23 2007 & 1 & - & \\
\hline & & & \\
\hline & & &
\end{tabular}

The exposure time was $1800 \mathrm{~s}$ with FEROS and $1200 \mathrm{~s}$ with NARVAL.

The log of observations is given in Table 1. FEROS observations $(R=48000,3600-9200 \AA)$ were reduced with MIDAS $^{2}$ (wavelength calibration, bias and flat field corrections and earth motion correction). For Narval observations $(R=$ $65000,3690-10480 \AA$ ), the reduction was first performed using the Libre-Esprit pipeline, based on ESPRIT (Donati et al. 1997). The intensity spectra extracted from each polarimetric sequence are used in the spectral analysis in this paper.

The determination of the continuum was done with several tasks of the IRAF sofware ${ }^{3}$.

The spectrum of HD 49330 is dominated by Balmer and He I lines. Lines of N II, O II, Si III and Mg II are also clearly present.

Fundamental parameters were previously determined by Frémat et al. (2006). They have been re-investigated and confirmed with the newly obtained high-resolution spectra using the same method as in Frémat et al. (2006). In Table 2, fundamental parameters of HD 49330 corrected for gravitational darkening effects at different values of $\Omega / \Omega_{\mathrm{c}}$ are given.

Spectral parameters and line profile variations $(l p v)$ have been studied. We find that circumstellar emission affects the photospheric lines to various degrees depending on their strength and the epochs in the observational run.

\footnotetext{
2 MIDAS is distributed by the European Southern Observatory (ESO). ${ }^{3}$ IRAF is distributed by the National Optical Astronomy Observatories, which is operated by the Association of Universities for Research in Astronomy (AURA), Inc., under cooperative agreement with the National Science Foundation.
} 
Table 2. Fundamental parameters of HD 49330 corrected for gravitational darkening effects at different $\Omega / \Omega_{\mathrm{c}}$ values.

\begin{tabular}{lllllllll}
\hline \hline$\Omega / \Omega_{\mathrm{c}}$ & $T_{\text {eff }}$ & $\log g$ & $\begin{array}{l}V \sin i \\
\mathrm{~km} \mathrm{~s}^{-1}\end{array}$ & $\begin{array}{l}i \\
\mathrm{deg}\end{array}$ & $\begin{array}{l}R_{\text {eq }} \\
R_{\odot}\end{array}$ & $\begin{array}{l}F_{\text {rot }} \\
\mathrm{c} \mathrm{d}^{-1}\end{array}$ & $\begin{array}{l}M \\
M_{\odot}\end{array}$ & $\begin{array}{l}L \\
L_{\odot}\end{array}$ \\
\hline 0.80 & 27416 & 3.82 & 279 & 58 & 8.62 & 0.78 & 14.27 & 4.47 \\
0.90 & 27964 & 3.87 & 281 & 43 & 8.70 & 0.96 & 14.40 & 4.46 \\
0.95 & 27432 & 3.82 & 286 & 40 & 9.63 & 0.92 & 14.28 & 4.47 \\
0.99 & 29000 & 3.94 & 290 & 33 & 9.08 & 1.18 & 14.87 & 4.47 \\
\hline
\end{tabular}

The errors bars on $T_{\text {eff }}$ are $\pm 1500 \mathrm{~K}$, on $\log g \pm 0.20$, on $V \sin i \pm$ $40 \mathrm{~km} \mathrm{~s}^{-1}$, on $i \pm 11 \mathrm{deg}$, on $R_{\mathrm{eq}} \pm 2.50 R_{\odot}$, on $F_{\text {rot }} \pm 0.35 \mathrm{c} \mathrm{d}^{-1}$, on $M \pm 0.30 M_{\odot}$ and on $L \pm 0.20 L_{\odot}$.

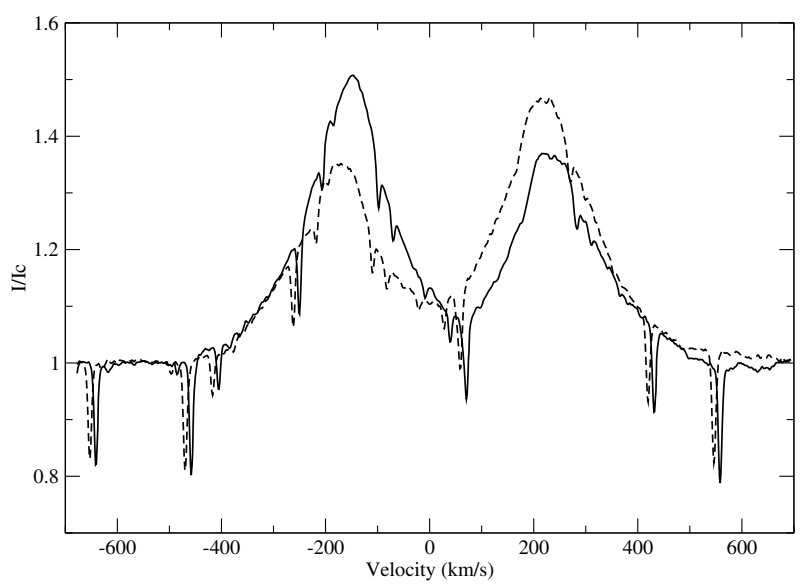

Fig. 2. Mean $\mathrm{H} \alpha$ line profiles in observed on 19-12-2007 (full line) and on 12-01-2008 (dashed line).

\section{Emission lines}

The $\mathrm{H} \alpha, \mathrm{H} \beta$ and red He I lines show a double-peaked emission ( $V$ and $R$ peaks) component with $V / R>1$ in the first part of the spectroscopic run (December 2007) and $V / R<1$ in the last part (January 2008) (see Fig. 2 for the $\mathrm{H} \alpha$ line).

\section{1. $\mathrm{Ho}$}

We measured the following characteristics of the $\mathrm{H} \alpha$ profiles: the intensity of the $V$ and $R$ emission components, the $V / R$ ratio, the peak separation $\Delta R V_{\text {peaks }}$ and the equivalent width of the global emission.

During the whole run the $V$ and $R$ emissions vary in an opposite way: in December 2007, the $V$ emission component strongly decreased while the $R$ component was stable, while in January 2008, at the end of the observing run, the $R$ component subtantially increased (see Fig. 3).

In spite of the gap in the observations between December 28, 2007 and January 10,2008 , the $V / R$ ratio is found to vary with a time span longer than 50 days (see Fig. 4).

Note that the $V / R$ ratio, as well as the stronger emission component ( $V$ peak), seems also modulated by a shorter timescale of about 10 days with a lower amplitude than the mid-term modulation (>50 d) reported above.

The equivalent width of the $\mathrm{H} \alpha$ emission $\left(E W_{\alpha}\right)$ contribution has been measured after subtracting the photospheric line profile centered at $+35 \mathrm{~km} \mathrm{~s}^{-1}$, which corresponds to the radial velocity of the star (see Sect. 4). In the data obtained in December 2007 we observe a 10-day modulation of low amplitude. In January $2008 E W_{\alpha}$ is lower than in December 2007 (see Fig. 5).

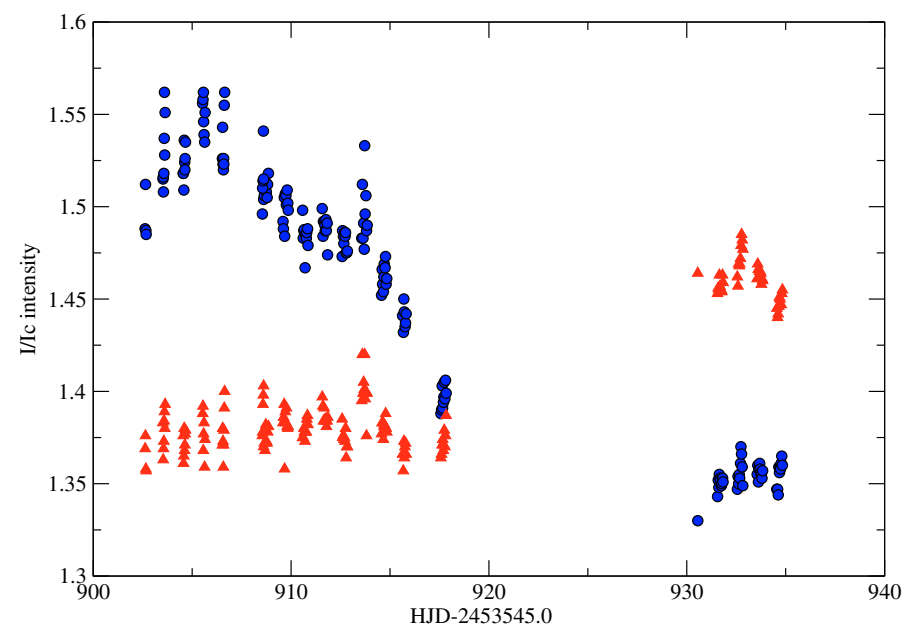

Fig. 3. Variation of the $\mathrm{H} \alpha$ emission peaks intensity of HD 49330. $V$ : filled blue circles; $R$ : filled red triangles.

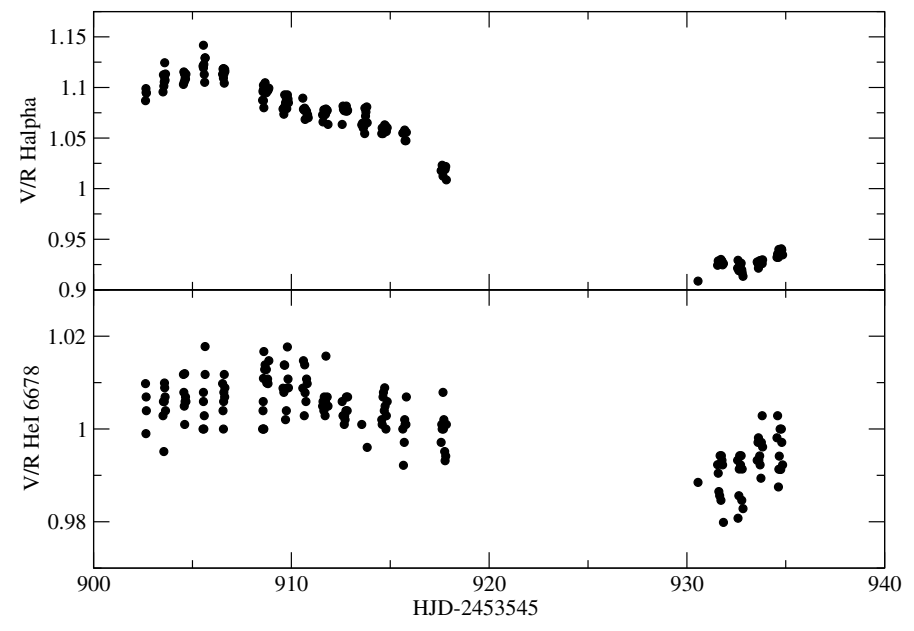

Fig. 4. $V / R$ variation of the emission peaks of $\mathrm{HD} 49330$ for the $\mathrm{H} \alpha$ (top) and He I 6678 (bottom) lines.

In summary, all the measured quantities in $\mathrm{H} \alpha$ vary over the timescale of the run and some of them seem, in addition, to be affected by a low amplitude modulation of about 10 days. Moreover, at CoRoT JD 2913 we note a small short-lived increase in the $V, R$ and $E W_{\alpha}$ quantities.

We have investigated the dimension of the disk from $\mathrm{H} \alpha$ and $\mathrm{H} \beta$ lines. As the wings of $\mathrm{H} \alpha$ are distorted by scattering (Poeckert \& Marlborough 1978; Rivinius et al. 2001a) their line width cannot be used to derive the inner edge of the emitting layers for this line. However, the peak separation provides an estimate of the radius of the maximum of emissivity of the disk for a given transition. It remains constant at about $380 \mathrm{~km} \mathrm{~s}^{-1}$. If we consider a roughly Keplerian law in the circumstellar disk following interferometric results (e.g. Meilland et al. 2007) and adopting $V_{\mathrm{kepl}}=530 \mathrm{~km} \mathrm{~s}^{-1}$ and $i \sim 52^{\circ}$ for a rotation rate $\sim 95 \%$ (Frémat et al. 2006), we find $R_{\text {peak }}=4.8 R_{*}$.

Similarly for $\mathrm{H} \beta$, with a peak separation of $460 \mathrm{~km} \mathrm{~s}^{-1}$, we find $R_{\text {peak }}=3.3 R_{*}$. We also derive the inner radius of the emitting layers for this line from its width $\left(850 \mathrm{~km} \mathrm{~s}^{-1}\right)$ and we conclude that the inner disk is close to the stellar surface. Thus there is no cavity between the star and the disk at the epochs of observation. 


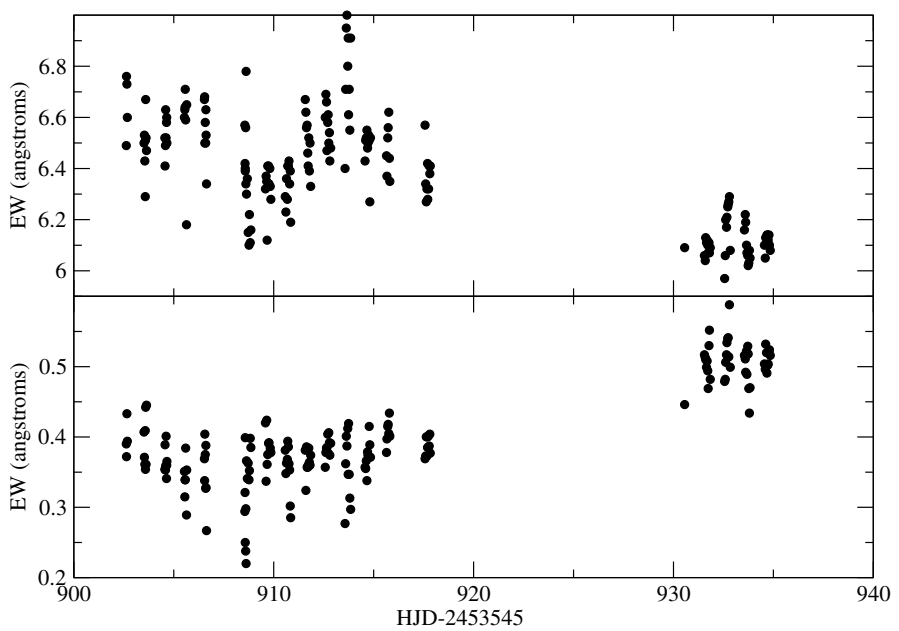

Fig. 5. Variation of the equivalent width of the $\mathrm{H} \alpha$ (top) and $\mathrm{He}$ I 6678 (bottom) emission of HD 49330 after subtraction of a photospheric synthetic profile centered at $+35 \mathrm{~km} \mathrm{~s}^{-1}$.

\section{2. $\mathrm{He} / 6678$}

The $V / R$ variation in the red He I lines is similar to the one in $\mathrm{H} \alpha$ (Fig. 4). However, the emission contribution in this line is more complex. This is seen by subtracting a synthetic photospheric line profile from each observed one. Then, the residual profiles show for most of the time a quadruple structure with a prominent $V$ peak in December 2007 and conversely a triple structure with a prominent $R$ one in January 2008 (see Fig. 6). Numerous DIBs listed in Weselak et al. (2000) are present in the spectra of HD 49330. Among them the one located at $6672.39 \AA$ strongly pollutes the blue emission component of He I $6678 \AA\left(-270 \mathrm{~km} \mathrm{~s}^{-1}\right.$ in Fig. 6). This DIB makes the study of the behaviour of the $V$ peak difficult, and consequently the separation of the $V$ and $R$ peaks, which is indicative of the changes in the disk extension. Note also the presence of a "central quasi-emission" (or CQE), already observed in other Be stars such as $\mu$ Cen (Rivinius et al. 1999). The origin of such a feature is controversial. The broad emission extends the line to the range $[-550,+600] \mathrm{km} \mathrm{s}^{-1}$, which is larger than the $[-V \sin i,+V \sin i]$ domain. Moreover, the width of the base of the line seems more extended between Dec. 19 and 28, 2007 (CoRoT JD 2909-2918) as the height of the more prominent $V$ peak drops gradually, showing some similarity with $\mu$ Cen within its precursor phase (Rivinius et al. 2001b). Finally, we note an increase by $35 \%$ of the equivalent width of the emission in the second set of FEROS data (Jan. 2008), compared to the first one (Dec. 2007, see Fig. 5). The multiple structure in the broad emission in He lines is probably indicative of successive ejecta, and the increase of the equivalent width supports the beginning of the outburst itself, depicted by the CoRoT data.

\section{Radial velocities}

We observed a shift in the radial velocities between December 2007 (Narval and the first part of FEROS run) and January 2008 (second part of FEROS run) of about $20 \mathrm{~km} \mathrm{~s}^{-1}$, for all the measured lines including blue He I and Si III lines. By subtracting the average night profile obtained on December 192007 from the one obtained on January 2008 (for example on January 132008 ) we can see that only the red wing

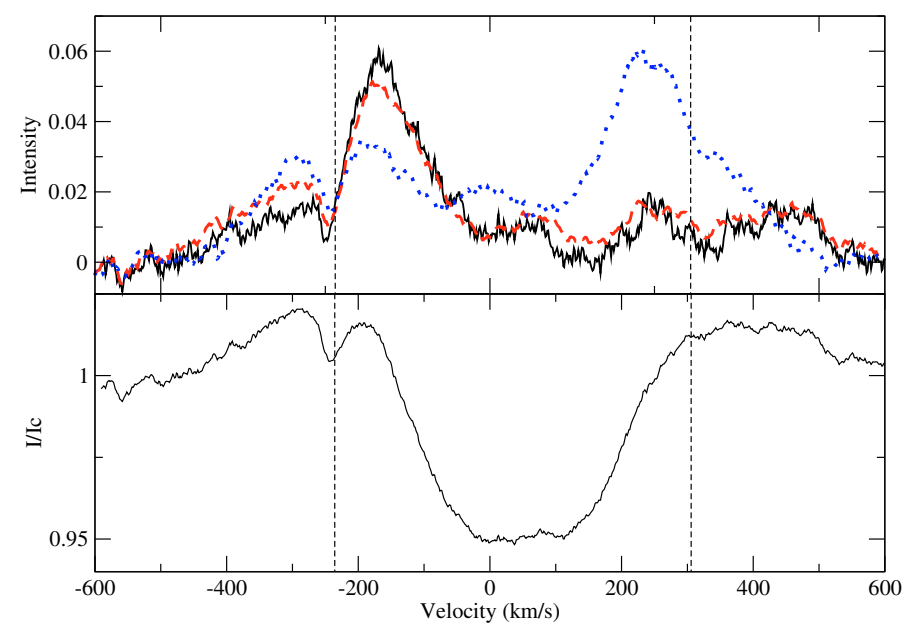

Fig. 6. Emission contribution for He I 6678 in HD 49330. Top: emission contribution obtained by subtraction of a photospheric synthetic profile from mean profiles of Narval observations (full black line), FEROS December observations (dashed red line) and FEROS January observations (dotted blue line). Bottom: mean line profile over the whole run. Dashed vertical lines indicate the $-V \sin i$ and $+V \sin i$ limits centered at $+35 \mathrm{~km} \mathrm{~s}^{-1}$.

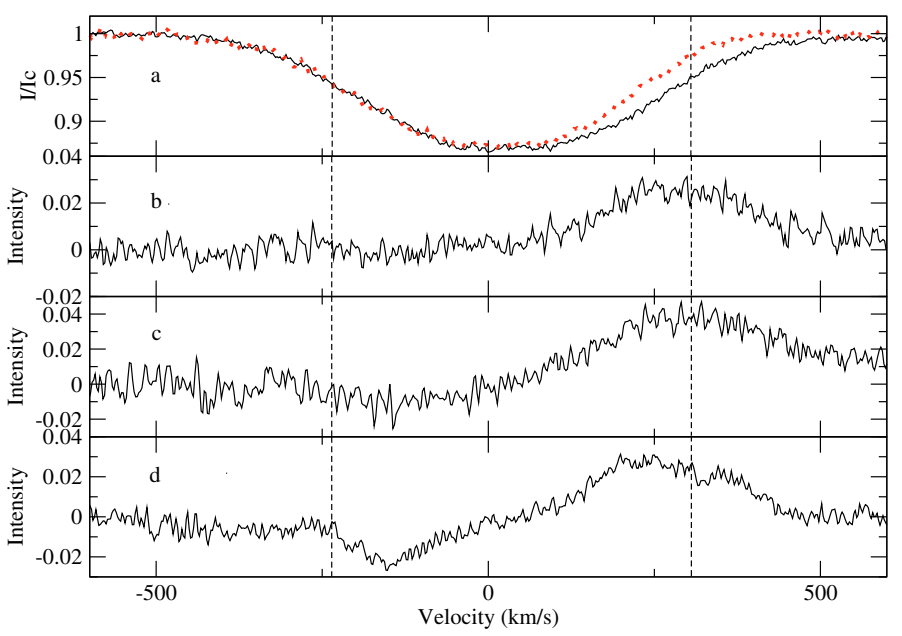

Fig. 7. Box a: He I 4026 mean line profiles observed on 19-12-2007 (full line) and on 12-01-2008 (dashed red line). Boxes b-d display the difference in the mean line profiles between the 12-01-2008 and 19-12-2007 data for the He I 4026, H I 3835 and He I 4921 lines, respectively. Dashed vertical lines indicate the $+V \sin i$ and $-V \sin i$ limits centered on $+35 \mathrm{~km} \mathrm{~s}^{-1}$.

of the line profile is polluted by emission (see Fig. 7) even for high Balmer lines. The position of this red emission structure (see Fig. 8) indicates an average velocity of $+360 \pm 50 \mathrm{~km} \mathrm{~s}^{-1}$ except for the red He I lines and first Balmer lines. For the latter lines, the additional emission structure is more complex, as it reflects the superposition of the red feature detected in all photospheric lines and the narrow component ( $R$ peak), which became stronger in January 2008.

The effective emitting layers in which the $V$ and $R$ peaks originate are more extended for the first Balmer lines than for the higher ones and thus the absolute value of the peak radial velocity depends on the line considered.

The blue He I, Si III and O II lines are less influenced by this emission especially in December 2007. However, O II lines 


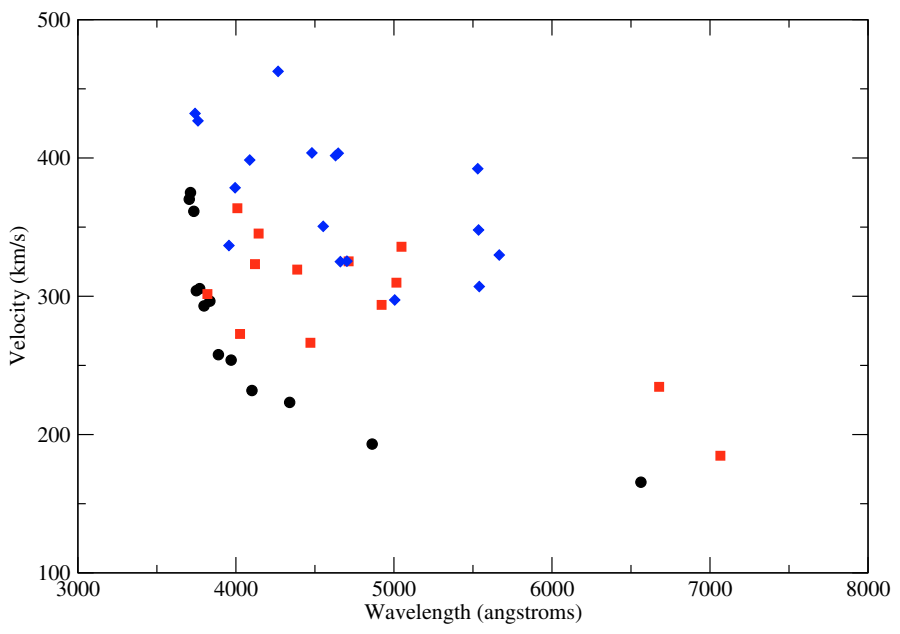

Fig. 8. Radial velocities of the red emission component observed in the line profiles: balmer lines (filled black circles), He I lines (filled red squares) and N II, Si III, Mg II lines (open blue diamonds).

suffer from severe blending and they could not be used for our purpose. On the other hand, according to Rivinius et al. (1999), the velocity variations in photospheric line centers have a stellar origin. Therefore, we measured the radial velocity of the centroid of the line core (typically at $I / I_{\mathrm{c}}=0.9$ ) of the He I 4026 and Si III 4553 lines in each spectrum. The daily fluctuations are about $30 \mathrm{~km} \mathrm{~s}^{-1}$ and the mean value is $\sim+35 \pm 3 \mathrm{~km} \mathrm{~s}^{-1}$.

Another method to determine the stellar radial velocity is the use of the radial velocity measurements of the wings of the $\mathrm{H} \alpha$ emission. This method is also robust for the determination of a true orbital RV curve, according to Harmanec et al. (2002). The radial velocity, measured on the bottom part (chosen at $I / I_{\mathrm{c}}=$ $1.075)$ of the $\mathrm{H} \alpha$ emission wings for each daily mean profile in December 2007 and January 2008 is $+35 \mathrm{~km} \mathrm{~s}^{-1} \pm 2 \mathrm{~km} \mathrm{~s}^{-1}$. This value is in good agreement with the one provided by the core of the blue He I lines in December 2007, when their profiles are less influenced by emission. The mean value $+35 \mathrm{~km} \mathrm{~s}^{-1}$ has thus been adopted for the stellar radial velocity of HD 49330 (see previous Sects. 3.1 and 3.2).

Thus the radial velocity variations observed in December 2007 and January 2008 cannot be due to an orbital motion since a global shift of the line would have been expected.

\section{Short-term variability}

The analysis of the CoRoT light curve obtained for HD 49330 has revealed the richness of its frequency spectrum (Huat et al. 2009). We search for frequencies in the high resolution, high signal-to-noise line profiles to compare them with the dominant frequencies detected by CoRoT and to help their mode identification. The main frequencies detected in the CoRoT light curve of HD 49330 are $11.86 \mathrm{c} \mathrm{d}^{-1}(137 \mu \mathrm{Hz}), 5.03 \mathrm{c} \mathrm{d}^{-1}(61 \mu \mathrm{Hz})$, $3.65 \mathrm{c} \mathrm{d}^{-1}(42 \mu \mathrm{Hz})$ and three groups of frequencies centered on $0.89 \mathrm{c} \mathrm{d}^{-1}(10 \mu \mathrm{Hz}), 1.47 \mathrm{c} \mathrm{d}^{-1}(17 \mu \mathrm{Hz})$ and $2.94 \mathrm{c} \mathrm{d}^{-1}$ (34 $\mu \mathrm{Hz}$ ). The amplitude associated with those frequencies has been found modulated over the run (Paper I). The analysis of the CoRoT data shows that the dominant frequencies are not the same in each part of the light curve. The $11.86 \mathrm{c} \mathrm{d}^{-1}$ frequency, dominant in the first part of the light curve (quiescent phase) has become gradually of weak amplitude, with a minimum during the third part (outburst itself), followed by a new increase during the relaxation phase. Conversely, the behaviour of the $0.89 \mathrm{c} \mathrm{d}^{-1}$, $1.47-1.57 \mathrm{c} \mathrm{d}^{-1}$ and $2.94 \mathrm{c} \mathrm{d}^{-1}$ frequencies is of very weak amplitude during the quiescent phase, the respective amplitude of these frequencies then gradually increased towards a maximum at the early stages of the light outburst. Fortunately, the second part of FEROS run (CoRoT JD 2930-2935) was carried out at the moment of constructive interference of amplitudes of the 1.47-1.57 and $2.94 \mathrm{c} \mathrm{d}^{-1}$ frequencies. Our results on the variability of the emission contribution in the spectral lines and especially between the first and the second parts of the spectroscopic run, suggest an outflow start, which prompted us to analyse separately the December 2007 and January 2008 observations.

The TSA is performed pixel by pixel on temporal series of line profiles and fitted on the whole profile with the least squares method (Kambe et al. 1993).

The accuracy on the determination of frequencies is $0.06 \mathrm{c} \mathrm{d}^{-1}$ in December 2007 and $0.20 \mathrm{c} \mathrm{d}^{-1}$ in January 2008. Taking into account the mean $S / N$ in December 2007 and in January 2008 we expect to detect a signal with amplitudes larger or equal to 4 and $4.5 \times 10^{-3}$ units of continuum, respectively.

In December 2007, all He I, Mg II, Si III and O II studied lines vary with two main frequencies: $f 1=11.86 \mathrm{c} \mathrm{d}^{-1}$ and $f 2=$ $16.89 \mathrm{c} \mathrm{d}^{-1}$. Their power and phase distributions are given in Fig. 9. In January 2008, the $16.89 \mathrm{c} \mathrm{d}^{-1}$ frequency is not detected in the line profiles and the amplitude of the $11.86 \mathrm{c} \mathrm{d}^{-1}$ is lower than in December 2007.

High frequencies are commonly observed in $\beta$ Cephei stars, and are thus expected in HD 49330 since it shares the same domain as this group of pulsating B stars in the HR diagram. Thus, we associate the two frequencies, $11.86 \mathrm{c} \mathrm{d}^{-1}$ and $16.89 \mathrm{c} \mathrm{d}^{-1}$, with non-radial pulsation p-modes. We use the method developed by Telting \& Schrijvers (1997) to derive the $\ell$ value of the corresponding modes. In that case their phase distribution indicates $\ell=4 \pm 1$ for $f 1$ and $\ell=6 \pm 1$ for $f 2$ (see Table 3 ). The sampling of the data and exposure time of each spectrum do not allow the detection of first harmonics of these frequencies and therefore the estimation of $|m|$ values. The best contrast is expected in the case of sectoral modes or in tesseral modes with $\ell-|m|=1$. However, as it has been underlined by Telting $\&$ Schrijvers (1997), their relation to derive $\ell$ and $|m|$ values were established for stars with moderate rotation. According to Lee (2007), the effects of rapid stellar rotation, which deform the star, strongly affect p-modes; much more elaborate models are required to reliably determine the modal properties of such modes in rapidly rotating stars.

In Fig. 10 the radial velocity of the He I 4026 core is folded in phase with the principal frequency $11.86 \mathrm{c} \mathrm{d}^{-1}$. The semi amplitude of the curve is about $10 \mathrm{~km} \mathrm{~s}^{-1}$ and the accuracy is $\pm 3 \mathrm{~km} \mathrm{~s}^{-1}$.

The $1.51 \mathrm{c} \mathrm{d}^{-1}$ frequency is detected in the data obtained in January 2008 but not in those of December 2007. In the CoRoT light curve the amplitude of the group of frequencies around $1.50 \mathrm{c} \mathrm{d}^{-1}$ has indeed become dominant at the epoch of the second part of spectroscopic observations. The anticorrelation between the amplitude variation of $f=11.86 \mathrm{c} \mathrm{d}^{-1}$ and $f=1.51 \mathrm{c} \mathrm{d}^{-1}$ is in agreement with what is observed in the CoRoT light curve (see Fig. 7 in Paper I).

Other main frequencies found in the CoRoT data $(2.94,3.65$ and $5.03 \mathrm{c} \mathrm{d}^{-1}$ ) are not detected in the present study of line profile variations. Note that the frequency $5.03 \mathrm{c} \mathrm{d}^{-1}$ is the exact difference between the two main spectroscopic frequencies $11.86 \mathrm{c} \mathrm{d}^{-1}$ and $16.89 \mathrm{c} \mathrm{d}^{-1}$. 

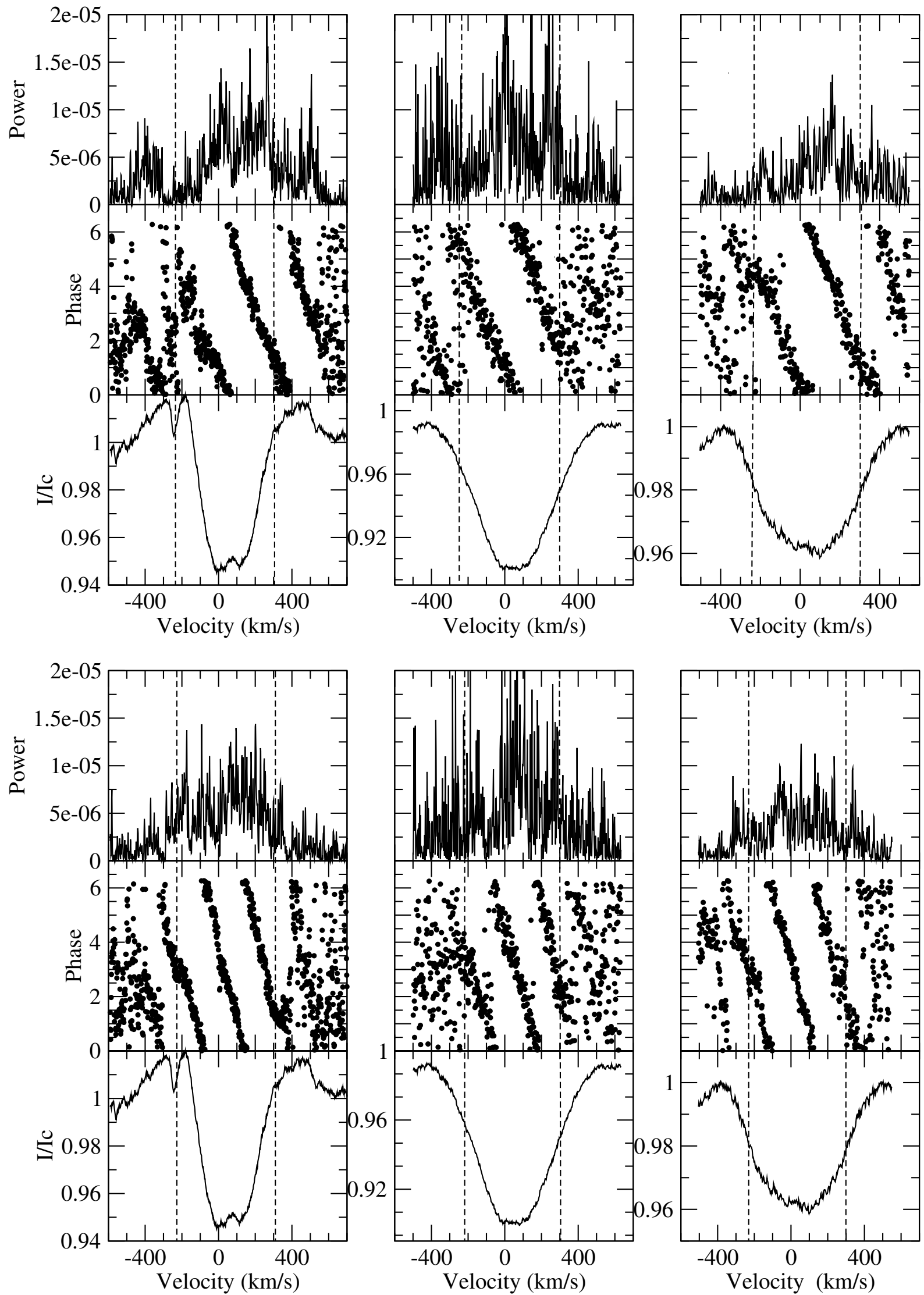

Fig. 9. Frequencies obtained from the TSA of photospheric line profiles: $f 1=11.86 \mathrm{c} \mathrm{d}^{-1}(137 \mu \mathrm{Hz})($ top $)$ and $f 2=16.89 \mathrm{c} \mathrm{d}{ }^{-1}(195 \mu \mathrm{Hz})($ bottom) for He I 6678 (left) and 4921 (middle) and Si III 4553 (right) in December 2007. Dashed vertical lines indicate the $+V \sin i$ and $-V \sin i$ limits centered on $+35 \mathrm{~km} \mathrm{~s}^{-1}$. The powers are expressed in units of continuum. 
Table 3. Amplitudes (in $10^{-3}$ units of continuum) for the main frequencies found in December 2007 and January 2008 in the He I 6678, 4921 and Si III 4553 lines.

\begin{tabular}{llllll}
\hline \hline $\begin{array}{l}\text { Frequency } \\
\mathrm{c} \mathrm{d}^{-1}(\mu \mathrm{Hz})\end{array}$ & $\begin{array}{l}\text { He I } \\
6678\end{array}$ & $\begin{array}{l}\text { He I } \\
4921\end{array}$ & $\begin{array}{l}\text { Si III } \\
4553\end{array}$ & $\begin{array}{l}\text { CoRoT } \\
\text { part 2 }\end{array}$ & Mode, $\ell$ \\
\hline $\begin{array}{l}\text { December 2007 } \\
11.86(137.26)\end{array}$ & 6.3 & 7.7 & 6.0 & 1.8 & $\mathrm{p}, 4$ \\
$16.89(195.48)$ & 5.6 & 6.9 & 5.3 & 0.35 & $\mathrm{p}, 6$ \\
$1.51(17.48)$ & - & - & - & 1.5 & - \\
\hline January 2008 & & & & part 3 & \\
$11.86(137.26)$ & 4.5 & 4.9 & 5.6 & 0.5 & $\mathrm{p}, 4$ \\
$16.89(195.48)$ & - & - & - & - & - \\
$1.51(17.48)$ & 8.0 & 7.7 & 6.3 & 1.9 & $\mathrm{~g} ?, 1-2 ?$ \\
\hline
\end{tabular}

Comparison with the amplitude found in the CoRoT light curve (in $10^{-3}$ units of flux) at the same epochs is given in Col. 5. Column 6 gives the expected type of mode and the estimate of $\ell$ deduced from the phase distribution along the line profile. The accuracy on the amplitudes is about $1.6 \times 10^{-3}$ and \pm 1 on the $\ell$ values.

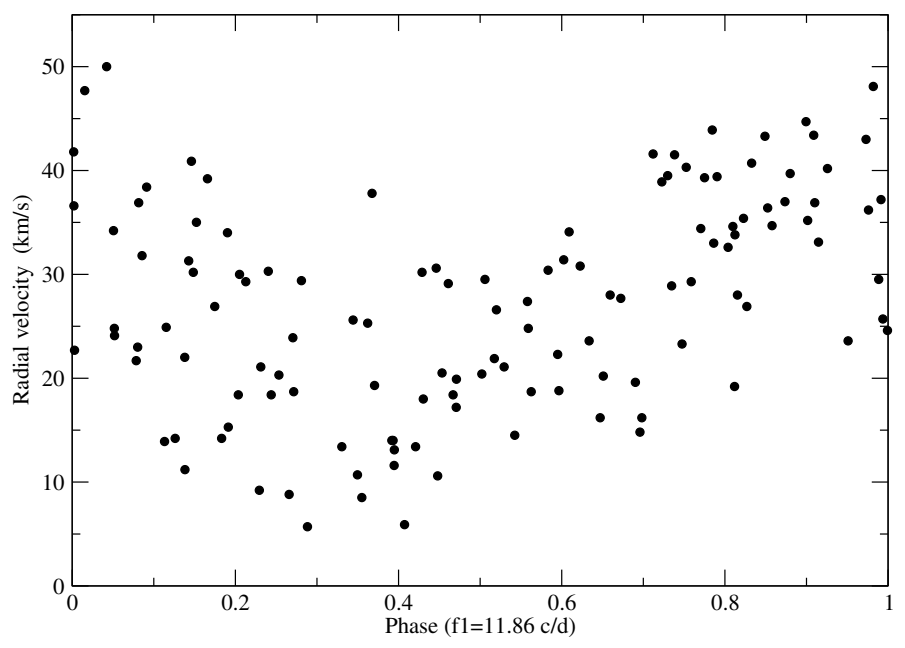

Fig. 10. Radial velocity of the core of He I 4026 obtained in December 2007 folded in phase with $f 1=11.86 \mathrm{c} \mathrm{d}^{-1}$.

\section{Discussion}

There is evidence of matter outflow in the spectroscopic data obtained in December 2007, near the end of the precursor phase of the light outburst depicted in the CoRoT data. As in $\mu$ Cen, at the epoch of an outburst, the total line width of He I emission (red lines) widely exceeds the quantity $2 V \sin i$, suggesting a rapidly rotating or moving gas close to the star. In January 2008, the equivalent width of $\mathrm{He}$ I emission, i.e. the quantity of material ejected at the stellar surface, has increased while the global intensity of the thick lines $(\mathrm{H} \alpha)$ has slightly decreased. According to Rivinius et al. (1998), ejecta could affect the recombination conditions by shielding the stellar radiation in some parts of the disk.

The broad emission feature detected at $+350 \pm 50 \mathrm{~km} \mathrm{~s}^{-1}$ in blue photospheric lines in January 2008 can be the signature of a non-symmetrical ejection. The change in the $V / R$ ratio observed in red He I lines and the first Balmer lines between December 2007 and January 2008 could be related to the appearance of this broad emission feature at $+350 \mathrm{~km} \mathrm{~s}^{-1}$. We propose that the change in the $V / R$ ratio observed in the red He I and first Balmer lines between December 2007 and January 2008 could be related to outflow episodes of material transfered from the stellar surface into an orbit at the base of the envelope. Mennickent \& Vogt (1991) detected $V / R$ changes on time-scale of $6-100 \mathrm{~d}$ in several Be stars and attributed them to the rotation of blobs in the circumstellar envelope which could result from a segmentation of ejected material.

The spectroscopic detection of high frequencies was expected in this very early Be star, which falls in the instability domain of $\beta$ Cephei stars in the H-R diagram. Sub-features rapidly crossing photospheric line profiles are associated with the high frequencies 11.86 and $16.89 \mathrm{c} \mathrm{d}^{-1}$; such regularly moving bumps have also been found from spectroscopy in other early Be stars or rapidly rotating $\mathrm{B}$ stars and attributed to high degree $(\ell>3)$ p-modes (see for instance HD 93521, Rauw et al. 2008; and $\zeta$ Oph, Kambe et al. 1997). The visibility of high degree modes is enhanced since fast rotation allows us to spatially resolve the velocity structure across the stellar disk. However the method we used to estimate $\ell$ and $|m|$ concerns stars with moderate rotation; it could be inaccurate or not valid for highly rotating stars. In addition, fast rotation favours the detection of high $\ell$ values due to coupling effects (Daszyńska-Daszkiewicz et al. 2002).

However, this is the first time that changes in the amplitudes have been detected in spectroscopy as well as in the light curve and have been associated with the presence of an outflow of matter. The amplitude fading for the high $11.86 \mathrm{c} \mathrm{d}^{-1}(137 \mu \mathrm{Hz})$ frequency, the vanishing of the other one at $16.89 \mathrm{c} \mathrm{d}^{-1}(195 \mu \mathrm{Hz})$, both associated with high degree p-modes, and the remarkable amplitude increase for the low $1.5 \mathrm{c} \mathrm{d}^{-1}(17 \mu \mathrm{Hz})$ frequency typical of g-modes observed in SPB, have to be related to the outflow episode revealed by this spectroscopic analysis as well as to the subsequent light outburst depicted by the CoRoT data.

\section{Conclusion}

Spectroscopic ground-based observations of the Be CoRoTLRA1 target HD 49330 have been obtained in the precursor phase just before the start of an outburst depicted in the CoRoT data. Time series analysis performed on photospheric line profiles allowed the detection of two main frequencies $f 1=$ $11.86 \mathrm{c} \mathrm{d}^{-1}$ and $f 2=16.89 \mathrm{c} \mathrm{d}^{-1}$. The first one is also the main frequency detected in the CoRoT light curve. These frequencies are attributed to high degree p-modes with $\ell=4$ and 6 , respectively, using relations for moderate rotators, in the absence of models appropriate for rapidly rotating stars. We showed that the stellar line profile variability has changed over the 32.2 day of observation in relation to the first stage of the outburst and is fairly in agreement with the CoRoT analysis.

Ejection of matter has been observed in the emission quantities at the epoch of minimum flux observed in the CoRoT light curve, just before the steep increase of brightness which characterizes the outburst itself, as commonly observed in Be stars. The ejection coincides with the constructive beating of the two main frequencies observed during the outburst phase of the CoRoT light curve, as shown in Paper I.

Acknowledgements. The FEROS data are being obtained as part of the ESO Large Programme: LP178.-0361 (PI: Poretti). This work was supported by the Italian ESS project, contract ASI/INAF I/015/07/0, WP03170. K.U. acknowledges financial support from a European Community Marie Curie IntraEuropean Fellowship, contract MEIF-CT-2006-024476. The CoRoT space mission, launched on December 27th 2006, has been developped and is operated by CNES, with the contribution of Austria, Belgium, Brasil, ESA, Germany and Spain. 


\section{References}

Daszyńska-Daszkiewicz, J., Dziembowski, W. A., Pamyatnykh, A. A., \& Goupil, M.-J. 2002, A\&A, 392, 151

Donati, J.-F., Semel, M., Carter, B. D., Rees, D. E., \& Collier Cameron, A. 1997, MNRAS, 291, 658

Frémat, Y., Neiner, C., Hubert, A.-M., et al. 2006, A\&A, 451, 1053

Gutiérrez-Soto, J., Fabregat, J., Suso, J., et al. 2007, A\&A, 476, 927

Harmanec, P., Bisikalo, D. V., Boyarchuk, A. A., \& Kuznetsov, O. A. 2002, A\&A, 396, 937

Huat, A.-L., Hubert, A.-M., Baudin, F., et al. 2009, A\&A, 506, 95

Hubert, A. M., \& Floquet, M. 1998, A\&A, 335, 565

Kambe, E., Ando, H., \& Hirata, R. 1993, A\&A, 273, 435
Kambe, E., Hirata, R., Ando, H., et al. 1997, ApJ, 481, 406

Lee, U. 2007, in Active OB-Stars: Laboratories for Stellare and Circumstellar

Physics, ed. A. T. Okazaki, S. P. Owocki, \& S. Stefl, ASP Conf. Ser., 361, 45 Meilland, A., Stee, P., Vannier, M., et al. 2007, A\&A, 464, 59

Mennickent, R. E., \& Vogt, N. 1991, A\&A, 241, 159

Poeckert, R., \& Marlborough, J. M. 1978, ApJS, 38, 229

Rauw, G., De Becker, M., van Winckel, H., et al. 2008, A\&A, 487, 659

Rivinius, T., Baade, D., Stefl, S., et al. 1998, A\&A, 333, 125

Rivinius, T., Štefl, S., \& Baade, D. 1999, A\&A, 348, 831

Rivinius, T., Baade, D., Štefl, S., \& Maintz, M. 2001a, A\&A, 379, 257

Rivinius, T., Baade, D., Štefl, S., et al. 2001b, A\&A, 369, 1058

Telting, J. H., \& Schrijvers, C. 1997, A\&A, 317, 723

Weselak, T., Schmidt, M., \& Krełowski, J. 2000, A\&AS, 142, 239 